\title{
APROXIMACIÓN AL ESTUDIO DE LA MORFOLOGÍA SETÍGERA EN ALGUNAS ESPECIES IBÉRICAS DE LOS GÉNEROS GLYCERA SAVIGNY, 1818 Y GLYCERELLA ARWIDSSON, 1899 (POLYCHAETA, GLYCERIDAE)
}

\author{
J. Parapar ${ }^{1} \&$ J. Moreira ${ }^{2}$
}

\section{RESUMEN}

J. Parapar \& J. Moreira. 2010. Aproximación al estudio de la morfología setígera en algunas especies ibéricas de los géneros Glycera Savigny, 1818 y Glycerella Arwidsson, 1899 (Polychaeta, Glyceridae). Grael/sia, 66(2): 119-130.

A partir del estudio al Microscopio Electrónico de Barrido (MEB) de las sedas espinígeras de varias especies del género Glycera Savigny, 1818 (Glycera alba Rathke, 1843; Glycera fallax Quatrefages, 1850; Glycera tesselata Grube, 1863; Glycera aff. tridactyla Schmarda, 1861 y Glycera unicornis Savigny, 1818) y Glycerella Arwidsson, 1899 (Glycerella magellanica (Mclntosh, 1885)), recogidas en la península lbérica, se aborda un estudio comparado preliminar de la morfología setígera a nivel de la zona de articulación entre el mango y el artejo. Como resultado de este estudio se han revelado diferencias en el número, tamaño y forma de los dientes que componen esta zona, lo cual apunta a un potencial valor de la microestructura setígera como carácter taxonómico a nivel subgenérico en la familia Glyceridae Grube, 1850, abriendo la posibilidad de su empleo en futuros estudios de revisión del grupo. Estas diferencias, si bien no serían susceptibles de ser empleadas en la identificación taxonómica rutinaria a nivel específico por no ser fácilmente observables sin el empleo del MEB, sí podrían ser utilizadas como recurso en futuros estudios sistemáticos y filogenéticos de la familia.

Palabras clave: Península Ibérica; Polychaeta; Glyceridae; Glycera; Glycerella; sedas.

\begin{abstract}
J. Parapar \& J. Moreira. 2010. An approach to the study of the chaetal morphology in some Iberian species of genus Glycera Savigny, 1818 and Glycerella Arwidsson, 1899 (Polychaeta, Glyceridae). Grael/sia, 66(2): 119-130 (in Spanish).

The spiniger chaetae of several Iberian species of the polychaete genera Glycera Savigny, 1818 and Glycerella Arwidsson, 1899, namely Glycera alba Rathke, 1843, Glycera fallax Quatrefages, 1850, Glycera tesselata Grube, 1863, Glycera aff. tridactyla Schmarda, 1861, Glycera unicornis Savigny, 1818 and Glycerella magellanica (Mclntosh, 1885), were compared and studied under the SEM, with particular attention to the joint between blade and shaft. This study showed differences in the number, size and shape of the teeth present
\end{abstract}

Departamento de Bioloxía Animal, Bioloxía Vexetal e Ecoloxía, Facultade de Ciencias, Universidade da Coruña, Alejandro de la Sota 1, E-15008 A Coruña, Spain. E-mail: jparapar@udc.es

2 Estación de Bioloxía Mariña da Graña, Universidade de Santiago de Compostela, Rúa da Ribeira 1, A Graña, E-15590 Ferrol, Spain. E-mail: juan.moreira@usc.es 


\begin{abstract}
at the joint; this fact points out the potential taxonomic value of the chaetal microstructure at the subgeneric level within the Glyceridae Grube, 1850, also representing a starting point for more detailed forthcoming studies. In addition, those differences might not be useful for operational taxonomic identification because of the need of the SEM but may represent an additional tool for studies on the systematics and phylogeny of glycerids.
\end{abstract}

Key words: Iberian Peninsula; Polychaeta; Glyceridae; Glycera; Glycerella; chaetae.

\section{Introducción}

Las sedas de poliquetos son formaciones cuticulares localizadas en los parápodos, desarrolladas a partir de folículos epidérmicos, y cuya distribución y morfología ha sido tradicionalmente empleada en taxonomía como caracteres diagnósticos para la identificación (Specht, 1988; Gardiner, 1992). Esto es así por la alta diversidad que habitualmente muestran tanto a nivel de taxones de alto rango (familias) como incluso entre inferiores (géneros y especies). Sin embargo, esta cualidad no presenta el mismo grado de incidencia en todas las familias, por lo que su relevancia no es igual en todas ellas. Así por ejemplo, entre los Phyllodocida, el orden que junto con los Eunicida conforma el gran clado Aciculata (sensu Rouse \& Fauchald, 1997), mientras que en ciertas familias su peso en la discriminación genérica y específica es muy alto (e. gr. Syllidae Grube, 1850 y Polynoidae Malmgren, 1867), en otras es sin embargo mucho menor, estando relegado a una consideración subordinada (e. gr. Nereididae Johnston, 1845, Hesionidae Sars, 1862, Phyllodocidae Williams, 1851, Glyceridae Grube, 1850, Goniadidae Kinberg, 1866, Nephtyidae Grube, 1850).

De la mano de la amplísima variedad estructural de las sedas en poliquetos, existe una rica terminología asociada a su forma: simples, compuestas, falcígeras, espinígeras, ganchos, espinas, ganchos encapuchados, capilares, limbadas, uncinos, paleas, etc. Esta terminología, que ha surgido de su estudio por medio del microscopio óptico (Hausen, 2005) está siendo actualmente revisada debido al empleo cada vez más extendido del microscopio electrónico de barrido (MEB), el cual gracias a su mayor resolución, está revelando multitud de nuevos detalles en su microestructura.

La familia Glyceridae está compuesta por tres géneros: Glycera Savigny, 1818, Glycerella Arwidsson, 1899 y Hemipodia Kinberg, 1865. Hemipodia se caracteriza por poseer parápodos unirrámeos con sedas simples y compuestas, mientras que en Glycera y Glycerella éstos son de naturaleza birrá- mea y están provistos de sedas simples capilares en el notópodo y compuestas espinígeras en el neurópodo en Glycera, mientras que en Glycerella estas últimas sedas neuropodiales pueden ser tanto espinígeras como falcígeras. Las sedas compuestas de Glycera están típicamente dotadas de un mango basal en el que se articula un largo artejo distal provisto de espinas pequeñas en una de sus caras.

En lo que concierne al género Glycera, trabajos recientes tanto de revisión general de la clase Polychaeta (Wilson, 2000; Pleijel, 2001) como de la familia Glyceridae (Böggemann, 2002), valoran a las sedas únicamente en la discriminación genérica. Así, Böggemann (2002), en su repaso de los caracteres taxonómicos empleados en la familia afirma que "The chaetae show such slight interspecific variation that they are only used for classification into genera". Por ello, los caracteres habitualmente empleados en su taxonomía son fundamentalmente el número de anillos del prostomio (Fig. 1A) y segmentos (Fig. 1D), forma del soporte mandibular (alerón), presencia/ausencia y posición de las branquias en los parápodos, forma de las papilas de la probóscide (Figs. 1B-C) y el tamaño relativo de las lengüetas pre y postsetales del parápodo (Figs. 1D, 6A) (Böggemann et al., 2000; Böggemann \& Fiege, 2001; Worsfold, 2007). Esta consideración de la quetotaxia en Glycera como un carácter sin relevancia taxonómica procede ya de trabajos clásicos como el de Fauvel (1923) en la costa francesa, Ushakov (1955) en los mares occidentales de la antigua Unión Soviética y Day (1967) en Sudáfrica. Trabajos más modernos de revisión de la fauna de diferentes áreas geográficas habitualmente continúan con esta tradición no ilustrando las sedas de las distintas especies o realizando dibujos poco precisos (e. gr. Gilbert, 1984; Lee, 1984; O'Connor, 1987; Böggemann \& Fiege, 2001; Kirkegaard, 2001; Jirkov, 2001; Böggemann \& Eibye-Jacobsen, 2002). Sin embargo, en este sentido es interesante destacar el trabajo de HartmannSchröder (1971) y su segunda edición realizada 25 años después (Hartmann-Schröder, 1996) en el cual 


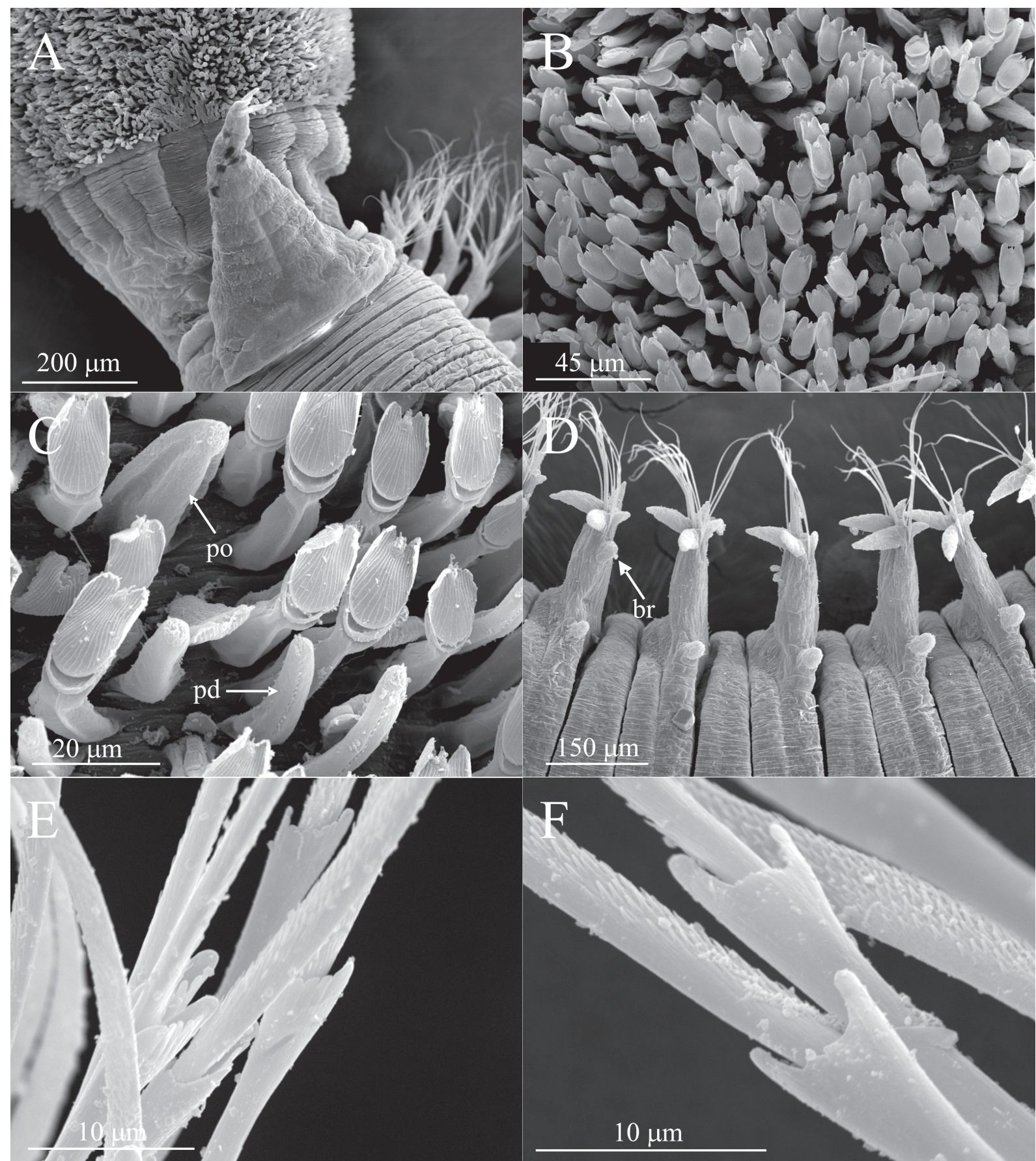

Fig. 1.- Glycera alba. A) Vista dorsal del prostomio y región anterior de la probóscide. B) Región de la probóscide cubierta de típicas papilas en forma de uña. C) Una papila oval y tres papilas digitiformes entre varias en forma de uña. D) Parápodos anteriores en vista dorsal, observándose en el último de ellos la aparición de la primera branquia. E-F) Articulación de las sedas espinígeras de los setígeros 16 y 26. br = branquia; pd = papila digitiforme; po = papila oval.

Fig. 1.- Glycera alba. A) Dorsal view of prostomium and anterior region of proboscis. B) Anterior region of proboscis covered with typical terminal fingernail papillae. C) One oval papilla and three digitiform papillae between fingernail papillae. D) Anterior parapodia in dorsal view, in the last one the first branchia is present. E-F) Joint of compound spinigers from chaetiger 16 and 26. br = branchia; pd = digitiform papilla; po = oval papilla. 
si bien la autora tampoco emplea las sedas como elemento taxonómico relevante en las descripciones, las ilustraciones que presenta son mucho más fieles a la realidad de los detalles de su arquitectura, pudiéndose apreciar algunos de los aspectos que a continuación comentaremos en las especies ibéricas mediante el empleo del MEB. Finalmente, Rizzo et al. (2007), en su reciente trabajo sobre las especies de esta familia en el litoral de Brasil, si bien tampoco dan gran valor taxonómico a las sedas compuestas, describen con mayor detalle que otros autores la forma de la zona articular y su variación dorso-ventral en el parápodo.

Al contrario que en Glyceridae, en otras familias de Aciculata con semejantes limitaciones en el empleo de la quetotaxia como herramienta taxonómica sí se han hecho intentos de revisión en este sentido. Por ejemplo, Eibye-Jacobsen (1991) realiza un detallado estudio de la zona de articulación de las sedas compuestas en diferentes géneros de Phyllodocidae, revelando ligeras pero importantes diferencias que aparentemente sí podrían ser empleadas tanto para la caracterización genérica como infragenérica en el caso de los géneros Eulalia Savigny, 1818 y Eumida Malmgren, 1865.

En la península Ibérica, el conocimiento de los glicéridos se podría calificar de bueno, especialmente a partir de la publicación de la monografía de Campoy (1982), la cual retomó el trabajo de Enrique Rioja tras su exilio de España por la Guerra Civil, y que representó en buena medida el punto de partida del estudio moderno de los poliquetos en nuestro país. La presencia habitual de estos poliquetos en fondos tanto someros como profundos de naturaleza arenosa y areno-fangosa los convierte en objeto de atención en numerosos estudios de ecología bentónica y taxonomía (e. gr. Rodríguez \& Viéitez, 1992; Parapar et al., 1993; Parra et al., 1995). Hasta el momento se han citado 10 especies de Glycera a lo largo de todo su litoral (Ariño, 1987; Parapar et al., 1996) así como recientemente también la presencia de la única especie reconocida del género Glycerella, Glycerella magellanica (McIntosh, 1885) en el talud continental gallego (Parapar \& Moreira, 2009).

Con ocasión del proyecto "Fauna Ibérica IX: Polychaeta IV", se ha tenido la posibilidad de estudiar un buen número de ejemplares de distintas especies ibéricas del género Glycera, obtenidas tanto de colecciones personales de diversos investigadores españoles, como a partir del material cedi- do por el Museo Nacional de Ciencias Naturales de Madrid (MNCN) procedente en buena medida de las campañas Fauna, así como también varios ejemplares del género monotípico Glycerella obtenidos en las campañas DIVA-Artabria I y II.

En este trabajo exploramos la variabilidad en la forma de esta zona de articulación de las sedas compuestas de Glycera y Glycerella, valorando su posible interés como carácter taxonómico a emplear en el futuro en el grupo. A continuación, presentamos las observaciones realizadas así como unos comentarios a las principales características anatómicas de las sedas de cada una de estas especies; ello podría ser de utilidad como base para un estudio más amplio de la quetotaxia de los glicéridos, el cual pudiera resultar, a su vez, en su empleo como un elemento más en el reconocimiento taxonómico de las especies así como para su estudio sistemático y filogenético.

\section{Material y Métodos}

Los ejemplares empleados para el examen de las sedas al MEB, uno por especie, han sido preparados en punto crítico, cubiertos por oro en un evaporador BAL-TEC SCD 004 y examinados y fotografiados en un MEB del tipo JEOL JSM-6400 en los Servicios de Apoio á Investigación (SAI) de la Universidade da Coruña (UDC). El material examinado se encuentra depositado en el Museo Nacional de Ciencias Naturales de Madrid (MNCN).

MATERIAL EXAMINADO: MNCN 16.01/13181: Glycera alba Rathke, 1843 (Campaña DIVA-Artabria 2002; 08/09/2002; plataforma gallega, $43^{\circ} 35.451^{\prime} \mathrm{N}$; $08^{\circ} 34.432$ 'W; 150 metros), MNCN 16.01/13183: Glycera fallax Quatrefages, 1850 (Colindres, Santander), MNCN 16.01/13184: Glycera tesselata Grube, 1863 (Nerja-Málaga; 15/09/1981; concreciones calcáreas), MNCN 16.01/13182: Glycera aff. tridactyla Schmarda, 1861 (ría de Ferrol; 11/08/1987; frente a punta Promontorio; 43⒉ 52 'N; $08^{\circ} 14.16^{\prime \prime W}$; 13 metros), MNCN 16.01/2557: Glycera unicornis Savigny, 1818 (Entre cabo San Antonio y puerto de Valencia), MNCN 16.01/11625: Glycerella magellanica (McIntosh, 1885) (Campaña DIVA-Artabria 2002; talud continental gallego;

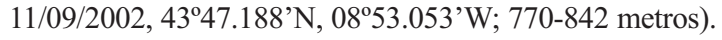

\section{Resultados y Discusión}

En las sedas compuestas del género Glycera, localizadas en la región neuropodial del parápodo, el elemento potencialmente relevante para la caracterización taxonómica se localiza en la zona de arti- 


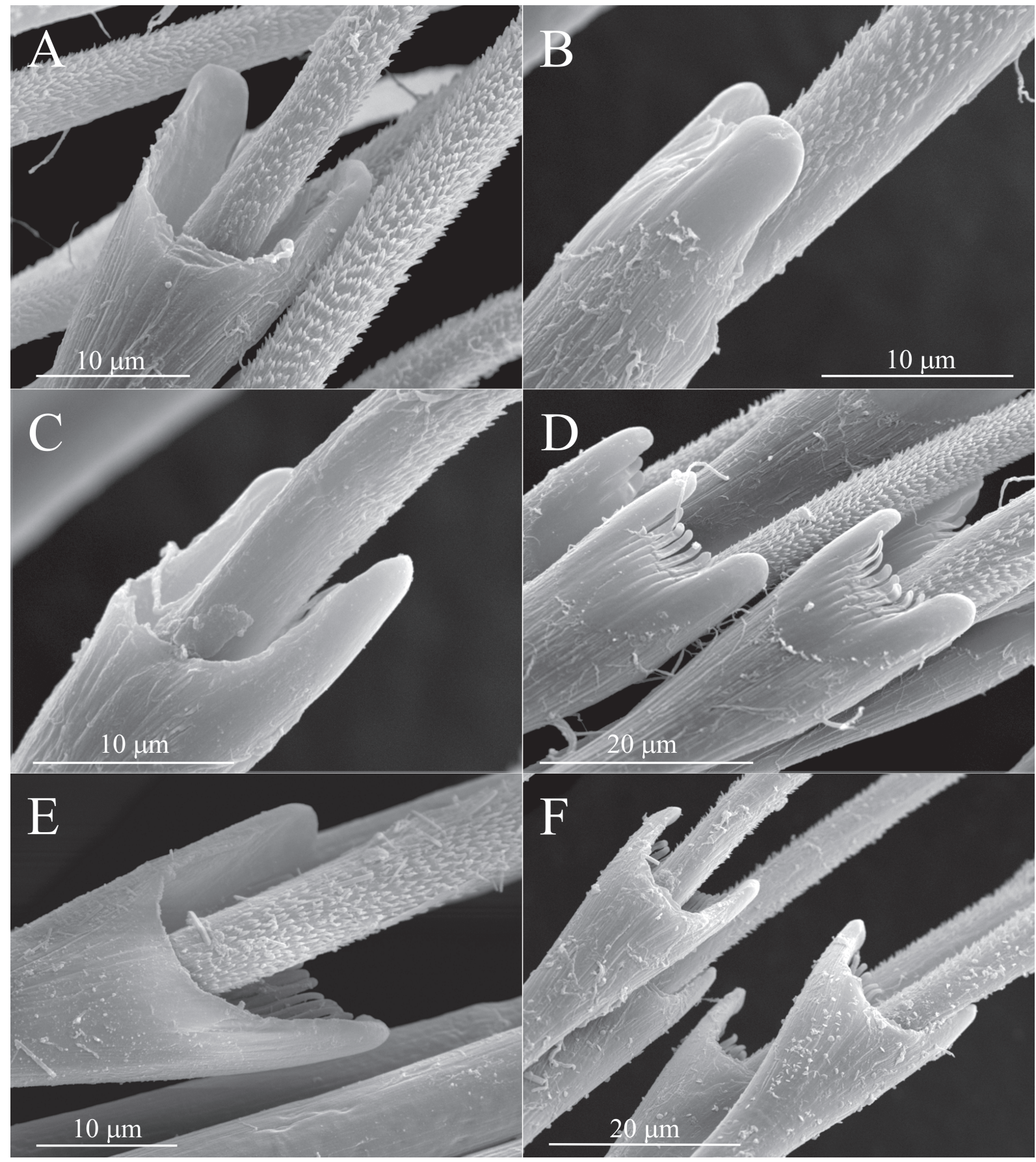

Fig. 2.- Glycera fallax. A-F) Sedas espinígeras. A-C) Setígero 79. D) Setígero 81. E) Setígero 152. F) Setígero 212.

Fig. 2.- Glycera fallax. A-F) Spiniger chaetae. A-C) Chaetiger 79. D) Chaetiger 81. E) Chaetiger 152. F) Chaetiger 212. 


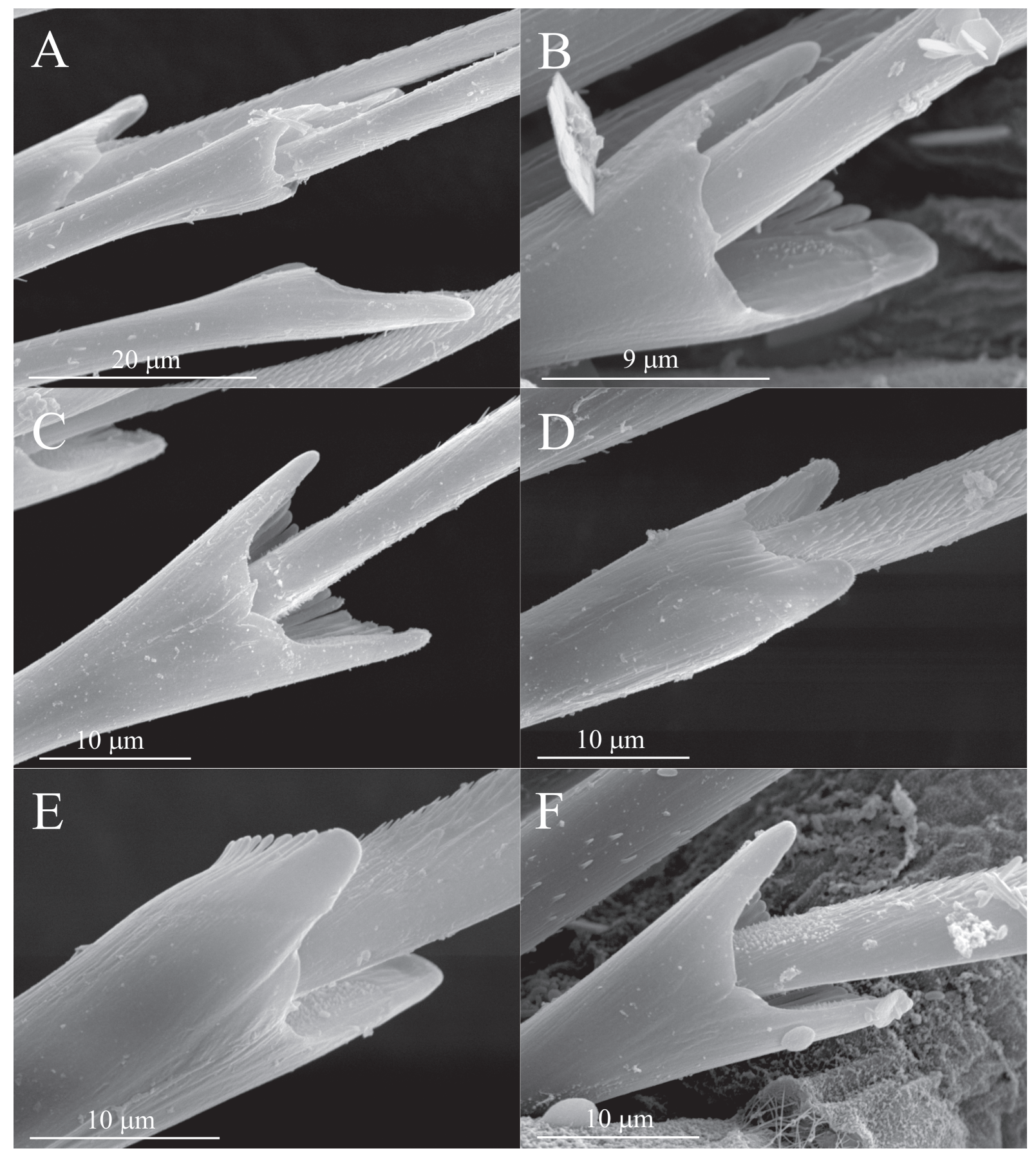

Fig 3.- Glycera tesselata. A-F) Sedas espinígeras. A) Setígero 9. B) Setígero 22. C-D) Setígero 33. E) Setígero 57. F. Setígero 69.

Fig 3.- Glycera tesselata. A-F) Spiniger chaetae. A) Chaetiger 9. B) Chaetiger 22. C-D) Chaetiger 33. E) Chaetiger 57. F) Chaetiger 69. 


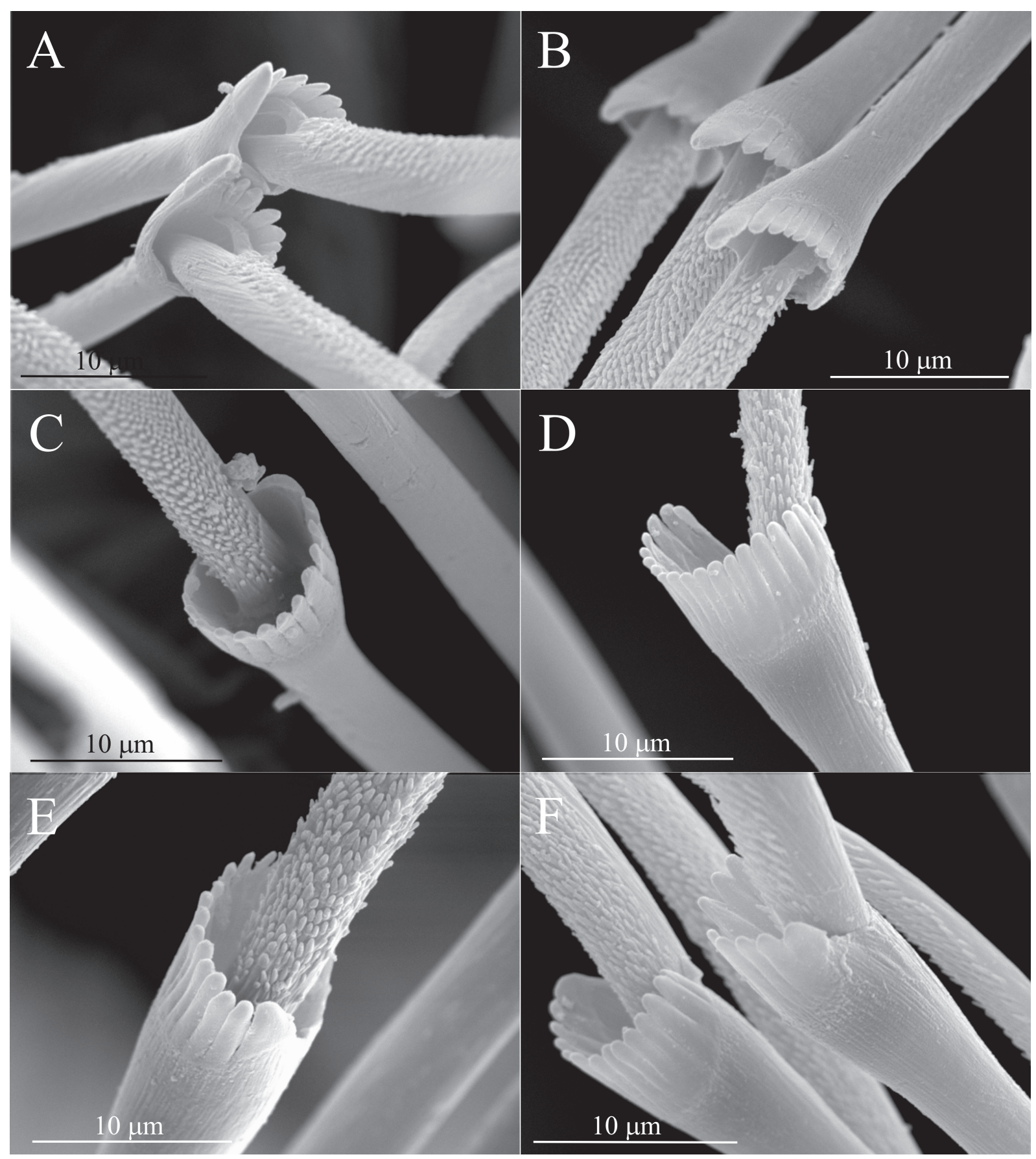

Fig. 4.- Glycera aff. tridactyla. A-F) Sedas espinígeras. A) Setígero 2. B) Setígero 5. C) Setígero 15. D) Setígero 18. E-F) Setígero medio.

Fig. 4.- Glycera aff. tridactyla. A-F) Spiniger chaetae. A. Chaetiger 2. B) Chaetiger 5. C) Chaetiger 15. D) Chaetiger 18. E-F) Midbody chaetiger. 


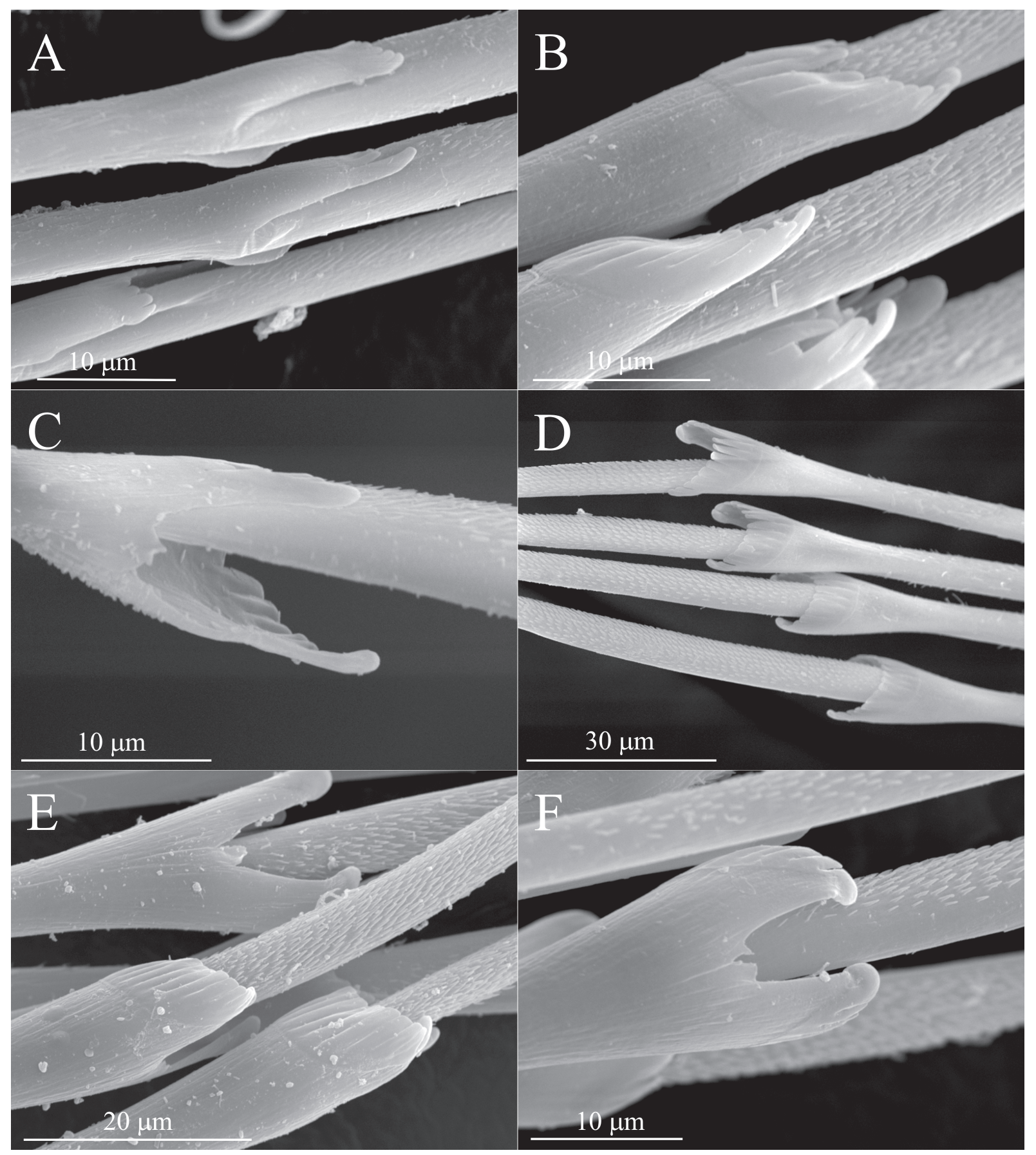

Fig. 5.- Glycera unicornis. A-F) Sedas espinígeras. A) Setígero 15. B) Setígero 37. C) Setígero 68. D) Setígero 84. E) Setígero 107. F) Setígero 175.

Fig. 5.- Glycera unicornis. A-F) Spiniger chaetae. A) Chaetiger 15. B) Chaetiger 37. C) Chaetiger 68. D) Chaetiger 84. E) Chaetiger 107. F) Chaetiger 175. 


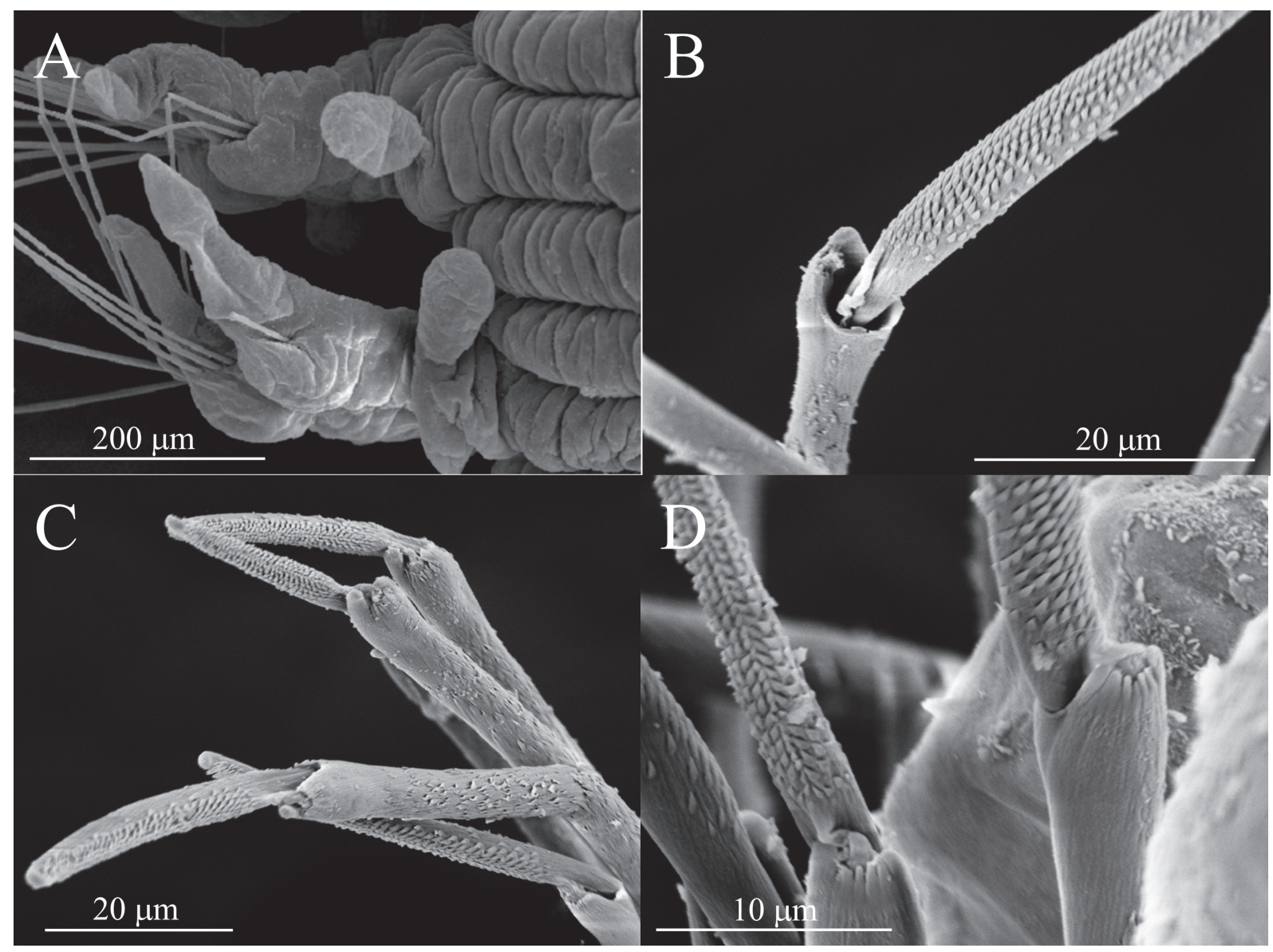

Fig. 6.- Glycerella magellanica. A) Setígero 15 (abajo) y 16 (arriba), vista posterior. B) Articulación de seda espinígera. C) Sedas falcígeras. D) Articulación de las sedas falcígeras.

Fig. 6.- Glycerella magellanica. A) Chaetiger 15 (below) and 16 (up), posterior view. B) Joint of spiniger chaeta. C) Falciger chaetae. D) Joint of falciger chaetae.

culación entre el mango y el artejo. En esta zona el mango está típicamente dotado de una profunda depresión cuyo borde posee forma de media luna, con una gran escotadura en su cara posterior, y una ornamentación en forma de dentículos parcial o totalmente fusionados en toda su longitud, en su cara anterior. En esta depresión en forma de embudo se inserta el extremo proximal del largo artejo distal (Fig. 4C), el cual se halla denticulado en una de sus caras que es la que habitualmente se orienta hacia la parte denticulada del mango (Figs. 4 D-F) (esto último no siempre es así; compárense por ejemplo las figuras $2 \mathrm{~A}$ y $2 \mathrm{E}$ frente a $2 \mathrm{D})$. Si atendemos al número de dientes de la cara anterior del mango y su grado de fusión, solamente en G. alba, con 4 dentículos centrales de pequeño tamaño (Fig. $1 \mathrm{E})$ se observa lo que parece ser una diferencia sustancial frente a las otras especies estudiadas: 8 en $G$. fallax (Fig. 2D) y 12-13 en G. tesselata, G. aff. tridactyla y G. unicornis (Figs. 3D, 4E-F y 5D respectivamente). No obstante, este hecho podría ser consecuencia del tamaño de las especies examinadas; en efecto, los ejemplares de G. alba han sido los de menor tamaño de todos los estudiados, debido, a su vez, al pequeño porte de esta especie (hasta $75 \mathrm{~mm}$ de longitud y 126 setígeros según Böggemann, 2002). Sin embargo, si atendemos al tamaño de los dientes extremos de la serie, se podrían definir tres grupos de especies. Así, mientras que en $G$. fallax y G. tesselata se observan unos dientes 
de gran tamaño flanqueando a unos dientes centrales claramente menores (véase Figs. 2B-D, 3B-F), en $G$. alba aquellos sólo son ligeramente mayores (Fig. 1E); por el contrario, en $G$. aff. tridactyla y $G$. unicornis los dientes externos son de un tamaño semejante a los centrales (Figs. 4D-F y 5C-E). Particularmente, en G. unicornis estos dientes parecen tener una forma peculiar que da a la cara posterior del mango una forma característica (Fig. 5F). Otras especies aquí no estudiadas podrían incluirse en uno u otro de estos grupos. Así, si atendemos a las ilustraciones de G. lapidum Quatrefages, 1865 de Hartmann-Schröder (1996: 242-243/figura 110), esta especie podría ser incluida en el primer grupo y G. rouxii Audouin \& Milne-Edwards, 1833 en el segundo (Hartmann-Schröder, 1996: 244-245/111). Las sedas que esta autora ilustra para G. alba y $G$. gigantea Quatrefages, 1866 ( $G$. fallax syn.) coinciden plenamente con las de los ejemplares ibéricos aquí examinados (Hartmann-Schröder, 1996: 240241/108 y 241-242/109, respectivamente).

Al igual que señalan Rizzo et al. (2007) para Hemipodia Kinberg, 1865, hemos constatado en Glycera la existencia de una clara modificación en la forma de las sedas de un mismo individuo en sentido antero-posterior; así, las sedas más próximas al prostomio son más sencillas y presentan una forma claramente diferente a las posteriores, con un seno mucho más marcado (véanse las Figs. 3A, 4AB y 5 A-B para $G$. tesselata, $G$. aff. tridactyla y $G$. unicornis, respectivamente).

A diferencia de lo ilustrado por HartmannSchröder (1996), los artejos no parecen articulados mediante estructura alguna en el fondo de la depresión central del mango (Figs. 4A, 4C), si bien esta observación podría ser un artefacto del MEB. En cualquier caso, parece existir una importante flexibilidad a ese nivel entre los dos componentes de la seda, la cual permite una amplia libertad de movimiento del artejo con respecto al mango, pudiendo el primero formar ángulos desde $0^{\circ}$ (Fig. 4E) hasta unos $70-80^{\circ}$ (Figs. 4A, 4D) con respecto al eje del segundo. Esta movilidad del artejo estará más limitada hacia la cara anterior de la seda por la menor flexibilidad de los dentículos del mango, mientras que será más amplia por su cara posterior por la presencia de la citada escotadura (Figs. 4D, 4F); los movimientos laterales por su parte estarían todavía mucho más limitados por la presencia de los dientes laterales del borde del mango, particularmente en las especies en las que éstos son gruesos (Fig. 2F).
Por su parte, las sedas compuestas del género Glycerella son claramente diferentes a las de Glycera. En Glycerella, las sedas compuestas neuropodiales son tanto falcígeras como espinígeras y en ellas tanto el mango como el artejo (Figs. 6B-C) están denticulados en una de sus caras y la zona de articulación entre ambos, particularmente en las falcígeras, está organizada de un modo diferente (Figs. 6C-D).

A modo de conclusión se podría afirmar que: a) la forma de las sedas compuestas espinígeras es claramente diferente entre Glycera y Glycerella confirmándose su utilidad como elemento diferenciador, b) parecen observarse diferencias en la forma de las sedas compuestas de las distintas especies estudiadas del género Glycera en la península Ibérica, las cuales se centran en la arquitectura de la zona de articulación entre el mango y el artejo, c) según el tamaño de los dientes extremos de la cara anterior del mango se podría explorar la elaboración de morfo-grupos de especies según su coincidencia en esta característica, d) el estudio de un elevado número de individuos de cada especie podría revelar la existencia de cierta variabilidad en la forma de las sedas, tanto en sentido antero-posterior (particularmente entre las sedas más anteriores y las del resto del cuerpo) como a nivel dorsoventral dentro de un mismo parápodo. Estas diferencias, si bien quizás no puedan emplearse en la identificación taxonómica rutinaria de especies por no ser de suficiente entidad ni fácilmente observables sin el empleo del MEB, sí podrían ser susceptibles de ser empleadas como recurso en el estudio sistemático y filogenético del género Glycera y quizás también para Hemipodia.

En definitiva, nuestros resultados apuntan a un potencial valor de la microestructura de las sedas de la familia Glyceridae como carácter taxonómico a nivel subgenérico, abriendo la posibilidad de su empleo en futuros estudios de revisión de la sistemática del género Glycera y filogenia general de la familia. De todos modos, estos resultados deben ser considerados como una primera aproximación a un estudio más amplio que incluya a otras especies del género.

\section{Agradecimientos}

Los autores desean expresar su agradecimiento a Pontxio Aguirrezabalaga (UPV-EHU), Julián Martínez (Sociedad Cultural INSUB, Donostia), Javier Murillo (IEO, Vigo), Javier Sánchez Almazán (MNCN, Madrid) y Santiago Parra (IEO, A 
Coruña) por la cesión de ejemplares de varias especies de Glycera. Queremos agradecer igualmente a Ada Castro y Catalina Sueiro (SAIN, UDC) por su ayuda en el empleo del MEB y a Santiago Parra y un revisor anónimo por sus acertados comentarios que han contribuido a la mejora de este manuscrito.

Este trabajo es una nueva contribución a los proyectos "Fauna Ibérica IX: Annelida Polychaeta IV: Lumbrineridae, Oenonidae y Glyceriformia" (CGL2007- 66786-C08-02) y "DIVA Artabria II" (CTM2004-00740/MAR) financiados por el Ministerio de Educación y Ciencia y "DIVA Artabria I" (PGIDT 01PXI20008PR) financiado por la Xunta de Galicia.

\section{Referencias}

Ariño, A., 1987. Bibliografía Ibérica de Poliquetos. Base de datos y catálogo de especies. Publicaciones de Biología de la Universidad de Navarra, Serie Zoológica, 16: 1-143.

Böggemann, M., 2002. Revision of the Glyceridae Grube, 1850 (Annelida: Polychaeta). Abhandlungen der Senckenbergischen Naturforschenden Gesellschaft, 555: 1-249.

Böggemann, M. \& Eibye-Jacobsen, D., 2002. The Glyceridae and Goniadidae (Annelida: Polychaeta) of the Bioshelf project, Andaman Sea, Thailand. Phuket Marine Biological Center Special Publication, 24: 149-196.

Böggemann, M. \& Fiege, D., 2001. Description of seven new species of the genus Glycera Savigny, 1818 (Annelida: Polychaeta: Glyceridae). Ophelia, 54 (1): 29-49.

Böggemann, M., Fiege, D. \& Purschke, G., 2000. Ultrastructure of the proboscidial papillae in some Glycera species (Annelida: Polychaeta: Glyceridae). Cahiers de Biologie Marine, 41: 143-153.

Campoy, A., 1982. Fauna de España. Fauna de Anélidos Poliquetos de la Península Ibérica. Ediciones de la Universidad de Navarra. Pamplona. 780 pp.

Day, J. H., 1967. A Monograph on the Polychaeta of Southern Africa. Part I Errantia. Trustees of the British Museum (Natural History). London. 458 pp.

Eibye-Jacobsen, D., 1991. Observations on setal morphology in the Phyllodocidae (Polychaeta: Annelida), with some taxonomic considerations. Bulletin of Marine Science, 48 (2): 530-543.

Fauvel, P., 1923. Polychètes Errantes. In: Faune de France, vol. V. Le Chevalier. Paris. 488 pp.

Gardiner, S. L. 1992. Polychaeta: External Anatomy. In: F. W. Harrison \& S. L. Gardiner (eds.). Microscopic Anatomy of Invertebrates, 7; Annelida, Willey-Liss, New York: 11-17.

Gilbert, K., 1984. Family Glyceridae Grube, 1850. In: M. Uebelacker \& P.G. Johnson (eds.). Taxonomic guide to the polychaetes of the northern Gulf of Mexico. J. Vittor \& Associates, Inc. Mobile, Alabama: 32-1 a 32-26.
Hartmann-Schröder, G., 1971. Annelida, Borstenwürmer, Polychaeta. In: Die Tierwelt Deutschands, vol. 58. $1^{\text {a }}$ edición. Gustav Fischer. Jena. 594 pp.

Hartmann-Schröder, G., 1996. Annelida, Borstenwürmer, Polychaeta. In: Die Tierwelt Deutschands, vol. 58. $2^{\mathrm{a}}$ edición. Gustav Fischer. Jena. 648 pp.

Hausen, H., 2005. Chaetae and chaetogenesis in polychaetes (Annelida). Hydrobiologia, 535/536: 37-52.

Jirkov, I., 2001. Polychaeta of the Arctic Ocean. YanusK. Moskva. 632 pp.

Kirkegaard, J. B., 2001. Polychaetes of the families Glyceridae, Goniadidae, and Nereididae from the North Atlantic around Iceland. Sarsia, 86: 13-20.

Lee, J. H., 1984. Polychaetous annelids from the Yellow Sea. II. Family Glyceridae. Bulletin of Kordi, 6: 1319.

O'Connor, B., 1987. The Glyceridae (Polychaeta) of the North Atlantic and Mediterranean, with descriptions of two new species. Journal of Natural History, 21: 167-189.

Parapar, J., O'Connor, B., Urgorri, V. \& Besteiro, C., 1993. Primera cita de Glycera dayi O'Connor, 1987 (Polychaeta, Glyceridae) en las costas ibéricas. Boletín de La Real Sociedad Española de Historia Natural, Sección Biológica, 90(1-4): 43-46.

Parapar, J., Besteiro, C. \& Urgorri, V., 1996. Inventario dos Poliquetos de Galicia (Annelida: Polychaeta). Cadernos da Area de Ciencias Biolóxicas. Publicación do Seminario de Estudos Galegos. 178 pp.

Parapar, J. \& Moreira, J., 2009. Sobre la presencia de Glycerella magellanica (McIntosh, 1885) (Polychaeta, Glyceridae) en el litoral de la península Ibérica. Graellsia, 65(2): 225-233.

Parra, S., Rodríguez, C. V., López-Jamar, E., O’Connor, B. D. S. \& Viéitez, J. M., 1995. Contribución al conocimiento del género Glycera Savigny, 1818 (Polychaeta: Glyceridae) en la península Ibérica. Clave taxonómica y nuevos datos sobre Glycera oxycephala Ehlers, 1887. Boletín del Instituto Español de Oceanografia, 11(1): 53-59.

Pleijel, F., 2001. Glyceriformia. In: G.W. Rouse F. y Pleijel (ed.). Polychaetes. Oxford University Press: 111-114.

Rizzo, A. E., Steiner, T. M. \& Amaral, A. C. Z., 2007. Glyceridae Grube, 1850 (Annelida: Polychaeta) from Southern and Southeastern Brazil, including a new species of Glycera. Biota Neotropica, 7 (3): 41-59.

Rodríguez, C. V. \& Viéitez, J. M. 1992. Macrofauna bentónica de los primeros metros del piso infralitoral de la costa de Punta Umbría (Huelva). Boletín del Instituto Español de Oceanografía, 8(2): 327 338.

Rouse, G. W. \& Fauchald, K., 1997. Cladistics and polychaetes. Zoologica Scripta. 26 (2): 139-204. 
Specht, A., 1988. Chaetae. In: W. Westheide \& C. O. Hermans (eds.). The Ultraestructure of Polychaeta. Microfauna Marina, 4: 45-59.

Ushakov, P. V., 1955. Polychaeta of the far eastern seas of the U.S.S.R. Academy of Sciences of the U.S.S.R. 419 pp. [Traducción realizada en 1965 del ruso al inglés por el Israel Program for Scientific Translations].

Wilson, R. S., 2000. Family Glyceridae. In: P.L. Beesley, G.J.B. Ross \& C.J. Glasby (eds.). Polychaetes \& Allies: The Southern Synthesis. Fauna of Australia, Vol. 4, Polychaeta, Myzostomida, Pogonophora, Echiura, Sipuncula. CSIRO Publishing. Melbourne: 127-129.

Worsfold, T. M., 2007. Identification guides for the NMBAQC Scheme: 2. Goniadidae with notes on
Glyceridae (Polychaeta) from shallow areas around the British Isles. Porcupine Marine Natural History Society Newsletter, 22: 19-23. Recibido / Received, 16-IV-2010 Publicado en línea / Published online, 20-X-2010 Publicado impreso / Published in print, 29-XII-2010 